\title{
Preface to the April 2018 Issue including selected works from CIbSE 2017 and LACLO 2016
}

\author{
Héctor Cancela ${ }^{1}$, Isabel Brito ${ }^{2}$, Luca Cernuzzi ${ }^{3}$, Marcela Genero ${ }^{4}$, Jesús García \\ Molina $^{5}$, Cristian Cechinel ${ }^{6}$ and Mario Chacón-Rivas ${ }^{7}$ \\ ${ }^{1}$ Universidad de la República, Uruguay, cancela@fing.edu.uy \\ ${ }^{2}$ Instituto Politécnico de Beja, Portugal, isabel.sofia@ipbeja.pt \\ ${ }^{3}$ Universidad Católica "Nuestra Señora de la Asunción", Paraguay, \\ lcernuzz@uc.edu.py \\ ${ }^{4}$ Universidad de Castilla-La Mancha, España, marcela.genero@uclm.es \\ ${ }^{5}$ Universidad de Murcia, España,jmolina@um.es \\ ${ }^{6}$ Universidade Federal de Santa Catarina - UFSC, Brazil, cristian.cechinel@ufsc.br \\ ${ }^{7}$ Instituto Tecnológico de Costa Rica, Costa Rica, machacon@itcr.ac.cr
}

This issue of the CLEIej consists of three main parts: i) a review paper on the state of the art of how contextual information extracted from a user task can help to improve searches for contents relevant to this task; ii) extended and revised versions of Selected Papers (which correspond to the second and third best paper from each track) presented at the XX Ibero-American Conference on Software Engineering (CIbSE 2017), which took place in Buenos Aires, Argentina, in May 2017; and, iii) extended and revised versions of selected papers from LACLO 2016, the XI Latin American Conference on Learning Objects and Technology, which took place in San José, Costa Rica, in October 2016.

The first paper included in this issue, "Searching in the Context of a Task: A Review of Methods and Tools", by Ana Maguitman, is an in-depth review of the state of the art of how to exploit topical context to identify the subject of the information needs related to a user task and satisfy specific information requests. Specifically, the article discusses different methods that have been proposed in the literature to solve the problem of reflecting current and long-term interests of a user in the search process, discussing the challenges of context-based information retrieval and presenting an overview of tools proposed since the mid-nineties to deal with the problem of contextbased search.

The second part, papers 2 through 6, focuses on the Selected papers from CIbSE 2017. CIbSE is the leading research forum on Software Engineering in IberoAmerica. The main objective of this conference is to promote high-quality scientific research in Ibero-American countries, supporting the researchers in this community in publishing and discussing their work. In addition, the conference fosters collaboration and cross-fertilization among software academics, students, and industry. CIbSE is organized in three Foundation Tracks: Software Engineering Track (SET); Experimental Software Engineering Latin American Workshop (ESELAW) and the Workshop on Requirements Engineering (WER). 
SET received 67 paper submissions from 24 countries. The Program Committee was composed by 72 experts from academia and industry from 16 countries. Each paper was revised by at least three members of the Program Committee and extensively discussed during an online discussion phase. At the end of the reviewing process, 24 high quality papers were accepted to be published and presented at the SET Track, representing an acceptance rate of $36 \%$.

ESELAW 2017 received 53 full paper submissions. The Program Committee was composed of 49 prestigious researchers from 16 countries. After a rigorous revision process, 19 high-quality scientific works were accepted from 10 countries, with an acceptance rate of $35.84 \%$.

WER received 38 abstract submissions, which were finally materialized in 33 full paper submissions from 10 countries. The Program Committee was composed of 40 experts from academia and industry from 13 countries. Each paper was reviewed by three members of the program committee and, after online discussion, 14 papers have been selected for presentation, which corresponds to a $42.42 \%$ of acceptation rate.

The two extended versions of the selected papers presented at SET 2017 are the following:

- Paper 2, by Helena Holmström Olsson and Jan Bosch, addresses the problem of modelling and measuring the customer value in software development. The authors have conducted a multi-case study research aimed to explore how value functions are defined in companies in the embedded system domain and the online domain, and they have discovered some weaknesses. This empirical evidence has been used to propose a comprehensive value function as the most beneficial one for companies across domains, and define a systematic approach to value modelling.

- Paper 3, by Constanza Pérez and Beatriz Marín, presents the open-source tool TCGen aimed at automating the generation of functional test cases from UML diagrams. TCGen automatically generates test cases using several testing criteria for the UML class diagram and for the UML state machine. A four-step strategy has been defined to generate the test cases: (1) generate the model, (2) identify structure and attributes of the models, (3) generate the abstract test cases, and (4) generate an executable script. The authors present an empirical evaluation of the tool, and the approach proposed is compared to traditional testing techniques.

The following papers were selected from ESELAW 2017:

- In Paper 4, Winkler et al. present a CrowdSourcing-based Inspection (CSI) process to support early defect detection of large-scale software engineering artifacts and models. The main goals of this inspection process are to improve defect detection effectiveness and to increase review artifact coverage. The authors present a controlled experiment involving 75 undergraduate students. The main goal of this experiment is to investigate the feasibility of applying the novel inspection process using a crowdsourcing tool and evaluate its effects compared to Pen \& Paper inspection process, considering medium size artifacts. This study showed that inspectors using CSI achieved results for defect detection 
effectiveness, which are comparable to the performance of inspectors using a traditional inspection process, and better defect detection efficiency.

- In Paper 5, Ribeiro and Travassos perform a literature review to identify source code attributes impacting the source code reading and comprehension. Moreover, they carried out an empirical study, with 38 undergraduate students, to support the assessment of four attributes (code size, length of identifiers, comments presence and indentation spacing) that presented empirical contradictions in the technical literature. After this thorough investigation, the authors suggested that further empirical research is required to explain the inconclusive results and to confirm others, to build a body of knowledge on which source code attributes influence source code comprehensibility and readability.

The last paper of this part of the issue was selected from WER 2017:

- In Paper 6, Wagner et al. build an empirical basis on such aspects of agile RE, based on the responses of representatives from 92 different organizations as part of a bigger survey initiative (Naming the Pain in Requirements Engineering). The survey data analyses revealed that agile RE concentrates on free-text documentation of requirements elicited with a variety of techniques. The backlog is the central means to deal with changing requirements. Commonly traces between requirements and code are explicitly managed and testing and RE are typically aligned. Furthermore, continuous improvement of RE is performed due to intrinsic motivation and RE standards are commonly practiced. Among the main problems, they highlight incomplete requirements, communication flaws and moving targets. Those problems were reported to happen commonly in agile projects and to have critical consequences, including project failure. Overall, their findings show that most organizations conduct RE in a way they would expect and that agile RE is in several aspects not so different from RE in other development processes.

The third part includes papers 7 and 8 of this issue, which are revised and extended versions of works selected from LACLO 2016. The Latin American Conference on Learning Technologies - LACLO has been taking place annually since 2006, and aims to be a forum where researchers, developers and education professionals can exchange experiences on several aspects of technological development applied to the context of learning. Through these years, the LACLO community has built an experience of recognizing the technology of learning objects from its definition and strengthening up to the encounter of more robust issues such as search and harvesting of learning objects, storage repositories, and most recently automatic recommendation, and initial usage of learning analytics associated with learning technologies. LACLO 2016 edition included 62 works from 12 different countries; the two extended versions of the selected papers presented at the conference are the following:

- In Paper 7, Camila Zacché de Aguiar, Davidson Cury and Amal Zouaq address a study of technological approaches for automatic construction of conceptual maps. They collected 30 articles published in relevant databases and categorized them based on data source and graphical representation, presenting characteristics and limitations for the created categories. This paper constitutes itself as a relevant reference related to the state of the art about automated construction of conceptual 
maps.

- In Paper 8, Roberto Munoz, Carlos Becerra, René Noël, Matías Camblor, Thiago S. Barcelos, Sandra V. Kreisel and Rodolfo Villarroel describe an user-centered design (UCD) experience to develop an app focused on supporting the teaching and learning process of children with autism spectrum disorders (ASD). The authors presented a documented introduction about the UCD and addressed important points of the APP development process. Finally, they describe the app validation based on data collected from 10 children diagnosed with ASD.

We would like to thank to the authors for delighting us with their high quality research and the members of the Program Committee of each track and conference for their effort and rigorous work done in the review process, as well as the CIbSE Steering Committee, the LACLO community and CLEIej for offering us the opportunity of preparing this special issue.

We are confident about the interesting contributions of this issue to the research and academic community.

Enjoy the reading!

Héctor Cancela, Isabel Brito, Luca Cernuzzi, Marcela Genero, Jesús García-Molina, Cristian Cechinel and Mario Chacón-Rivas, special issue editors 